\title{
NASCIDOS PARA CORRER: A IMPORTÂNCIA DO EXERCÍCIO PARA A SAÚDE DO CÉREBRO
}

\author{
BORN TO RUN: THE IMPORTANCE OF EXERCISE FOR THE BRAIN HEALTH
}

NACIDOS PARA CORRER: LA IMPORTANCIA DEL EJERCICIO PARA LA SALUD DEL CEREBRO

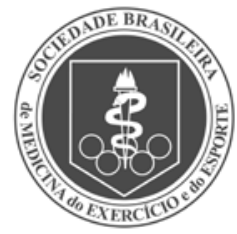

Artigo de Revisão Sistemática

Systematic Review Article

Artículo de ReVISIÓN SISTEMÁtICA

Camila Vorkapic-Ferreira (Psicóloga e Profissional de Educação Física)

Rayanne Souza Góis²

(Profissional de Educação Física)

Luis Paulo Gomes ${ }^{1}$

(Profissional de Educação Física)

Andre Britto $^{3}$

(Psicólogo e Publicitário)

Bastos Afrânio ${ }^{4}$

(Profissional de Educação Física)

Estélio Henrique Martins Dantas

(Profissional de Educação Física)

1. Universidade Tiradentes, Departamento de Educação Física e Psicologia, Laboratório de Biociências da Motricidade Humana - LABIMH, Farolândia, Aracaju, SE, Brasil.

2. Universidade Federal de Sergipe, Departamento de Fisioterapia, Laboratório de Neurociências, São Cristóvão, SE, Brasil.

3. Universidade Estácio de Sergipe, Departamento de Psicologia,

Aracaju, SE, Brasil.

4. Universidade Federal de Sergipe, Departamento de Educação Física,

São Cristóvão, SE, Brasil.

\section{Correspondência:}

Universidade Tiradentes Av. Murilo Dantas, 300, Farolândia, Aracaju, SE, Brasil. 49032-490. cfvorkapic@gmail.com

\section{RESUMO}

A hipótese evolutiva da corrida de resistência afirma que o movimento teve um papel crucial no aparecimento de características anatômicas tipicamente humanas, assim como na modelação da estrutura e forma do cérebro humano. A íntima ligação entre exercício e evolução humana é evidenciada pelo fato de a inatividade nos tornar doentes. Efetivamente, o corpo humano, incluindo o cérebro, evoluiu para suportar períodos prolongados de estresse cardiovascular. O movimento é de tal modo essencial para o cérebro, que a atividade física regular é imprescindível para que funcione de modo adequado. Estudos vêm demonstrando que o exercício aeróbico aumenta a proliferação de neurônios, a síntese de fatores neurotróficos, gliogênese, sinaptogênese, regula sistemas de neurotransmissão e neuromodulação, além de reduzir a inflamação sistêmica. Todos esses efeitos têm impacto significativo no sentido de melhorar a saúde mental, reduzir o declínio de massa cinzenta associado à idade e melhorar as funções cognitivas. Deste modo, o objetivo deste artigo é apresentar uma atualização sobre a temática de exercício físico e saúde mental. Dados os recentes avanços apresentados neste original, sobre a neurobiologia do exercício e seu potencial terapêutico e econômico para a população em geral, espera-se que pesquisas futuras que correlacionem estudos básicos a variáveis psicológicas e estudos de imagem possam elucidar os mecanismos pelos quais o exercício melhora a saúde cerebral.

Descritores: exercício; neurociência; saúde mental.

\section{ABSTRACT}

The evolutionary hypothesis of endurance running states that movement played a crucial role in the emergence of typically human anatomical features, as well as in the shaping and structure of the human brain. The close relationship between exercise and human evolution is evidenced by the fact that inactivity make us sick. Effectively, the human body, including the brain, has evolved to withstand extended periods of cardiovascular stress. Movement is so essential to the brain that regular physical activity is imperative for it to function properly. Studies have shown that aerobic exercise increases neuron proliferation, neurotrophic factors synthesis, gliogenesis, synaptogenesis, regulates neurotransmission and neuromodulation systems, and reduce systemic inflammation. All of these effects have a significant impact on improving mental health, reducing age-related gray matter decline, and improving cognitive functions. Thus, the purpose of this article is to present an update on the subject of physical exercise and mental health. Given the recent advances presented in this manuscript on the neurobiology of exercise and its therapeutic and economic potential for the general population, it is expected that future research that correlate basic studies with psychological variables and imaging studies may elucidate the mechanisms by which exercise improves brain health.

Keywords: exercise; neuroscience; mental health.

\section{RESUMEN}

La hipótesis evolutiva de la carrera de resistencia afirma que el movimiento tuvo un papel crucial en la aparición de características anatómicas típicamente humanas, así como en el modelado de la estructura y forma del cerebro humano. La íntima conexión entre ejercicio y evolución humana es evidenciada por el hecho de que la inactividad nos hace enfermos. De hecho, el cuerpo humano, incluido el cerebro, ha evolucionado para soportar períodos prolongados de estrés cardiovascular. El movimiento es tan esencial para el cerebro, que la actividad física regular es imprescindible para que funcione adecuadamente. Los estudios vienen demostrando que el ejercicio aeróbico aumenta la proliferación de neuronas, la sintesis de factores neurotróficos, gliogénesis, sinaptogénesis, regula sistemas de neurotransmisión y neuromodulación, además de reducir la inflamación sistémica. Todos estos efectos tienen un impacto significativo en la mejora de la salud mental, reducir la declinación de materia gris asociada a la edad y mejorar las funciones cognitivas. De este modo, el objetivo del artículo es presentar una 
actualización sobre el tema ejercicio físico y salud mental. De acuerdo con los recientes avances presentados en este original, sobre la neurobiología del ejercicio y su potencial terapéutico y económico para la población en general, se espera que investigaciones futuras que correlacionen estudios básicos a variables psicológicas y a estudios de imagen puedan esclarecer los mecanismos por los cuales el ejercicio mejora la salud del cerebro.

Descriptores: ejercicio; neurociencia; salud mental.

\section{INTRODUÇÃO}

Em 2004 foi sugerido que humanos haviam evoluído de ancestrais parecidos com macacos, especificamente devido à sua capacidade de correr longas distâncias. Segundo os autores ${ }^{1}$, a forte seleção para a corrida foi crucial na modelagem do corpo do homem moderno, sendo exatamente a habilidade em correr, um fator crucial para o aparecimento de características anatômicas específicas. De acordo com Lieberman², a corrida nos tornou humanos, pelo menos no sentido anatômico, e o aparcecimento dos humanos está intimamente ligado à evolução da corrida. A evolução de humanóides, como os Australopithecus, ao gênero Homo ocorreu há cerca de 2 milhões de anos como consequência da seleção natural, favorecendo a perpetuação de características anatômicas humanas que tornaram possível a corrida de longa distância, segundo os autores. Isso aconteceu porque, provavelmente, tais caraterísticas tornaram possíveis a fuga de predadores e a perseguição da caça, muito antes que o advento de arcos, flechas e redes reduzissem a necessidade de correr longas distâncias ${ }^{3}$. Segundo os autores, a importância da corrida na evolução humana é tal, que se a seleção natural não a tivesse favorecido, ainda pareceríamos macacos.

Mas, quais são esses traços humanos que tornaram a corrida possível? Dentre as características únicas e tipicamente humanas, estão: tendões dos pés que funcionam como molas; estrutura de pé e dedos que permite um uso propulsor eficiente; ombros que fazem rotação independentemente da cabeça e do pescoço, permitindo equilíbrio durante a corrida; um ligamento que se estende da nuca até as vértebras torácicas e atua como absorvente de choque à medida que ajuda os braços a contrabalancearem com o peso da cabeça durante a corrida (ligamento nucal); presença de grandes glúteos, massas musculares críticas para a estabilização do tronco especificamente durante a corrida; corpos longos; pernas alongadas, entre outras características tipicamente humanas ${ }^{4}$ (Figura 1). Os autores afirmam que apesar de hoje a corrida de resistência (endurance running) ser uma forma de recreação e exercício, sua raiz pode ser tão antiga quanto o aparecimento do gênero humano e suas demandas, um fator significativo na forma e fisiologia do corpo humano.

Deste modo, o que está implícito na hipótese da corrida de resistência é que o corpo humano evoluiu para suportar longos períodos de estresse cardiovascular, adaptando-se a um estilo de vida extremamente ativo, que pode ter direcionado o crescimento do cérebro há 2 milhões de anos. Como consequência, o movimento teve um papel crucial na modelagem da estrutura e forma do cérebro humano ${ }^{4}$. A possibilidade da ligação íntima entre exercício e evolução humana é sugerida pelo fato de a inatividade nos tornar doentes, física e mentalmente ${ }^{5}$. Na verdade, no que diz respeito ao sistema nervoso, estudos vêm demonstrando que o movimento é tão essencial para humanos que o cérebro, não só precisa, mas requer atividade física regular para funcionar de modo adequado ${ }^{6}$.
Nascidos para correr

a importância do exercício para a saúde do cérebro

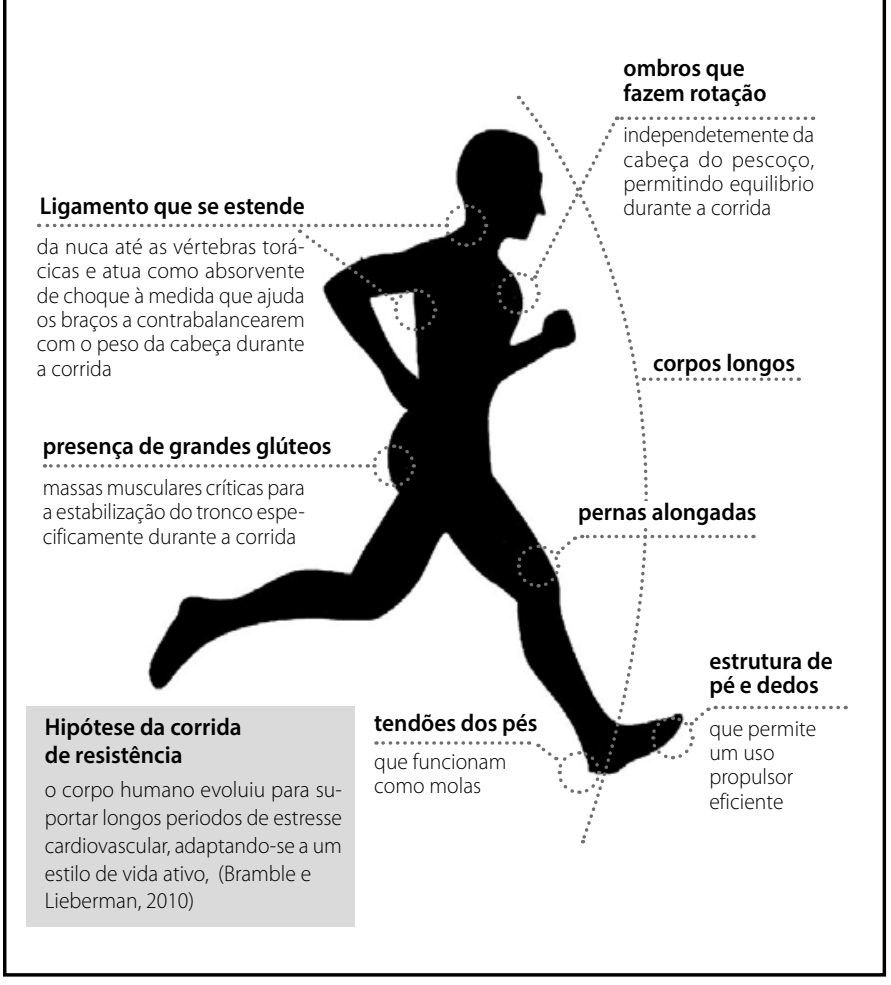

Figura 1. Segunda a teoria da corrida de resistência, características anatômicas e fisiológicas tipicamente humanas foram uma adaptação à corrida.

De fato, décadas de estudo vêm demonstrando o efeito significativamente positivo do exercício no cérebro, evidenciando a relação ancestral entre estresse cardiovascular (exercício) e saúde mental? Uma revisão ${ }^{8}$ sugeriu também que o exercício aeróbico regular reduz sintomas associados a diferentes transtornos mentais e doenças neurodegenerativas, como Alzheimer e Parkinson. Os autores concluíram que o exercício aeróbico pode ser usado como intervenção adjunta no tratamento de transtornos mentais. Outros estudos demonstraram que altos níveis de atividade física estão associados a níveis mais elevados de qualidade de vida entre indivíduos saudáveis e acometidos por diferentes transtornos mentais 9 . Sugere-se que os efeitos do exercício aeróbico no bem estar mental sejam consequência da regulação para baixo do eixo hipotalâmico-pituitário-adrenal (HPA) ${ }^{10}$. Paradoxalmente, o exercício é um fator estressor, mas apresenta efeito neuroprotetor. Estudos mostram que indivíduos submetidos a um programa de exercícios apresentam níveis mais baixos de cortisol durante repouso ou submetidos a um fator estressor, quando comparados a sedentários ${ }^{10}$. Estima-se que a regulação para baixo do eixo HPA pelo exercício aeróbico aconteça à partir de mecanismos de feedback negativo, através de um aumento na densidade e eficiência de mineralocorticóides e inibição de cortisol ${ }^{10}$. 
O exercício aeróbico também tem sido relacionado à melhoras cognitivas em jovens e idosos ${ }^{11,12}$ concluíram que uma melhor aptidão física está associada a melhors funções executivas e processos visuoespaciais. Estudos recentes propuseram que o exercício aeróbico protege o cérebro contra a demência ou retarda o declínio cognitivo relacionado à idade ${ }^{12}$.

Os estudos que sugerem que o exercício tem impacto nas funções cerebrais têm focado primariamente nos efeitos biológicos diretos, utilizando modelos animais e humanos. Mas, de acordo com Lista e Sorrentino ${ }^{11}$, o exercício também pode melhorar a cognição através da melhora geral em condições de saúde e da redução dos sintomas das doenças crônicas que têm impacto em funções neurocognitivas. A maioria das evidências acerca dos efeitos do exercício no cérebro, no entanto, vem de estudos em animais ${ }^{11-16}$ sugerem que os mecanismos neurobiológicos básicos associados ao exercício podem ocorrer em dois níveis: no nível extracelular, o exercício induz a angiogênese à partir de vasos pré-existentes. No nível intracelular, o exercício tem sido associado à neurogênese hipocampal ${ }^{11}$. O significado funcional desse efeito ainda é incerto, mas alguns estudos propõem que neurônios recém-formados podem ser totalmente integrados à rede neural, tornando-se funcionais ${ }^{14}$. O exercício parece ainda induzir o crescimento de novas sinapses (sinaptogênese) ${ }^{14}$. Além disso, estudos em animais mostraram alterações induzidas pelo exercício em fatores moleculares de crescimento como o fator neurotrófico derivado do cérebro (BDNF) e fator de crescimento semelhante à insulina tipo (IGF-1), proteínas com papel crucial na neuroplasticidade, neuroproteção e neurogêne$\mathrm{se}^{10}$. Há ainda vasta evidência que os sistemas de neuromodulação e neurotransmissão sejam modulados pelo exercício ${ }^{17,18}$.

Por último, um conceito emergente sugere que a saúde cerebral e as funções cognitivas são moduladas pela interrelação entre fatores centrais e periféricos ${ }^{19}$. Processos inflamatórios sistêmicos, presentes em doenças metabólicas como hipertensão arterial ou resistência à insulina, aumentam a inflamação no sistema nervoso central e estão associados ao declínio cognitivo ${ }^{19}$.

Em suma, décadas de estudos vêm demonstrando que os efeitos do exercício no cérebro são únicos no sentido de melhorar a saúde mental, funções cognitivas e reduzir fatores periféricos de risco que parecem influenciar diretamente a saúde do cérebro. Dados os recentes avanços na presente temática, o objetivo deste artigo de perspectiva é fornecer uma atualização da literatura no que concerne exercício e saúde mental. O presente manuscrito enfatiza, deste modo, os vários efeitos do exercício no cérebro de humanos e animais, como as alterações nas funções cerebrais, neuroquímica, sistemas de transmissão e gênese de novas células nervosas, assim como na relação entre estas mudanças e saúde mental. Sendo assim, este artigo se divide em duas partes: (1) Alterações neurofisiológicas e neuroquímicas após o exercício aeróbico em estudos humanos e animais e (2) a relação entre estas alterações e melhoras na saúde mental.

\section{Alterações neurofisiológicas e neuroquímicas após o exer- cício aeróbico}

\section{Mudanças bioquímicas e no eixo HPA}

A ativação do eixo hipotalâmico-pituitário-adrenal (HPA), envolvido na regulação de hormônios do estresse, como o cortisol, parece ter papel fundamental no efeito do exercício no cérebro. Quando estimulado, o hipotálamo libera o hormônio liberador de corticotrofina (CRH), que por sua vez estimula a glândula pituitária a sintetizar o hormônio adrenocorticotrófico. Este último interage com a glândula adrenal promovendo a síntese do cortisol (corticosterona em animais) ${ }^{20,21}$. Paradoxalmente, apesar do exercício agudo ser um agente estressor, o exercício crônico tem efeito neuroprotetor. Estudos mostram que indivíduos submetidos a um programa de exercícios apresentam menores níveis de cortisol em repouso ou em resposta a um agente estressor, quando comparados a sedentários ${ }^{10}$. Algumas hipóteses sugerem que alterações na atividade do eixo HPA, como maior densidade e eficiência de receptores mineralocorticóides, menores níveis de cortisol e inibição da síntese de cortisol, podem representar mecanismos eficientes de feedback negativo ${ }^{20}$.

Além disso, estudos em animais e humanos mostraram que alteraçõess hormonais podem influenciar comportamentos e funções alimentares através da interação com fatores anorexigênicos, como glicose e leptina, e fatores orexigênicos, como neuropeptídeo Y e grelina. Estes fatores podem regular o circuito alimentar no núcleo ventrolateral do hipotálamo, ativando o sistema em situações de estresse. Consequentemente, uma combinação de ingestão de aminoácidos e ativação do eixo HPA pode fazer com que o corpo guarde energia para situações estressantes ${ }^{20}$

Outros estudos observaram que o exercício crônico tem efeitos antioxidantes, que podem ser explicados pela sinalização mediada por espécies reativas de oxigênio (ROS). A produção mitocondrial de ROS, resultante de alta demanda metabólica, induz a sinalização mediada pelo fator de transcrição nuclear kappa B (NF-kB). Este induz a expressão de genes que codificam enzimas antioxidantes como a superóxido dismutase e a glutationa peroxidase, que combatem o acúmulo de radicais livres ${ }^{22}$. Além disso, o aumento na concentração de ROS modula a atividade de vias intracelulares envolvidas no comportamento de fibras musculares. Estudos animais observaram que maiores níveis de ROS ativam a proteína CREB (CAMP response element-binding) e o receptor ativado por proliferadores de peroxissoma gama (PGC-1a) no núcleo, induzindo a biogênese mitocondrial. Deste modo, o exercício aeróbico crônico promove um aumento da atividade biogênica mitocondrial (expressão dos fatores de respiração nuclear, NRF-1 e NRF-2, e em conseqüência, ativação do fator de transcrição mitocondrial A -TFAM) mediada por antioxidantes ${ }^{22}$.

\section{Sistemas de neurotransmissão e neuromodulação}

Evidências mostram que o exercício agudo promove alterações cerebrais em consequência do aumento no metabolismo, oxigenação e fluxo sanguíneo no cérebro. No entanto, a maioria das evidências disponíveis provém de pesquisas em animais ${ }^{11}$. Estes estudos mostram que o exercício agudo modula a maioria dos neurotransmissores no sistema nervoso central associados à inibição e sedação (GABA), estado de alerta (norepinefrina), sistema de recompensa (dopamina) e humor (serotonina) $)^{23,24}$. A ativação das monoaminas pela atividade física reduz a incidência e aumenta as chances de recuperação de transtornos mentais como depressão, ansiedade e estresse ${ }^{24}$. Nesse contexto, é interessante o fato de que agonistas de serotonina, incluindo alguns antidepressivos como a fluoxetina, podem aumentar a gênese celular25-27, enquanto a administração de antagonistas de receptores de serotonina reduzem a proliferação celular no giro denteado, uma região do hipocampo associada à potenciação de longo prazo ${ }^{27}$. De fato, os efeitos antidepressivos do exercício em humanos ${ }^{24,28,29}$ têm se mostrado tão potentes quanto o de medicações agonistas de serotonina, aumentando a possibilidade de a neurogênese ser o mecanismo comum terapêutico por trás das melhoras nos sintomas. Análises de varredura mostraram que tanto o exercício agudo quanto o crônico afetam a expressão de genes hipocampais associados à plasticidade sináptica de uma forma geral ${ }^{30}$. Mais especificamente, genes relacionados ao sistema glutamatérgico 
são regulados para cima e aqueles associados ao sistema GABAérgico, para baixo ${ }^{29}$. Na verdade, a função glutamatérgica no giro denteado pode regular a neurogênese ${ }^{29}$. As alterações na função glutamatérgica induzidas pelo exercício podem desse modo influenciar a produção e função de novos neurônios no cérebro adulto. No entanto, é improvável que esse aumento de ativação resulte em excitotoxidade associada ao glutamato, já que o exercício também eleva os níveis de proteínas neuroprotetoras como o BDNF²5.

Outros fatores neuroquímicos liberados durante o exercício agudo incluem o aumento na síntese de opióides e endocanabinóides, responsáveis pela sensação de euforia, bem-estar, sedação e redução à sensibilidade da dor ${ }^{31-37}$. Além disso, agonistas externos exógenos, como a morfina e a heroína, suprimem a neurogênese in vivo ${ }^{31,32}$, enquanto, endorfinas e encefalinas estimulam a gênese celular in vitro ${ }^{33-35}$. Os complexos efeitos dos opióides na produção de novos neurônios, no entanto, ainda permanecem incertos.

Outros estudos em animais mostraram ainda que o sistema endocanabinóide pode ter um papel relevante na sensação de sedação e bem estar após o exercício, conhecida como "onda de corredor" ${ }^{\prime \prime 37}$. As endorfinas não atravessam a barreira hematoencefálica, mas a molécula lipossolúvel da anandamida, um endocanabinóide, pode entrar no cérebro e desencadear as conhecidas sensações. Os autores fornecem uma visão completa de como esse importante sistema de recompensa está envolvido na melhora do estado psicológico e na sensibilidade à dor, em consequência do exercício.

\section{Fatores neurotróficos}

Fatores neurotróficos, proteínas essenciais para sobrevivência, proliferação e maturação neuronal, também são ativados e sintetizados durante o exercício agudo. Estudos em animais mostram aumento nos níveis de expressão de diversas neurotrofinas como o fator neurotrófico derivado do cérebro (BDNF), fator de crescimento semelhante à insulina tipo 1 (IGF-1), fator de crescimento vascular endotelial (VEGF), neurotrofina-3 (NT3), fator de crescimento de fibroblasto (FGF-2), fator neurotrófico derivado da glia (GDNF), fator de crescimento epidérmico (EGF) e fator de crescimento nervoso (NGF), após exercício ${ }^{38,39}$.

Nas últimas décadas, vem crescendo o interesse na relação entre fatores angiogênicos e neurogênese. No giro denteado, no hipocampo, os novos neurônios se aglomeram próximos aos vasos sangíneos e se proliferam em resposta aos fatores vasculares, como VEGF e IGF-138,39. Isto levou à hipótese de que células neurais progenitoras estão associadas a um nicho vascular e que a neurogênese e a angiogênese estão intimamente relacionadas ${ }^{40}$.

Em particular, estudos mostraram que a expressão do gene hipocampal de VEGF em animais adultos resulta em aproximadamente o dobro do número de neurônios no giro denteado e melhoras na cognição ${ }^{41-44}$. Além disso, a infusão periférica de IGF-1 também aumenta a neurogênese no cérebro de animais adultos, além de reverter a redução neuronal relacionada ao envelhecimento ${ }^{45}$. Deste modo, muitos autores concluem que as alterações vasculares no cérebro em consequência do exercício podem ser mediadas por fatores como o IGF e VEGF ${ }^{44-49}$.

Os efeitos de longo prazo do exercício parecem resultar de diferentes respostas e adaptações, comparados com os efeitos do exercício agudo. Uma série de alterações neuroqúímicas, como o aumento na expressão de fatores neurotróficos e a indução de processos anti-inflamatórios que promovem angiogênese, neurogênese e sinaptogênese, advém do aumento no fluxo sanguíneo cerebral em decorrência do exercício crônico $^{38}$. Apesar da maioria dos estudos serem realizados em animais, alguns destes resultados já foram extrapolados com sucesso para humanos, já que os mecanismos adjacentes apresentam respostas muito similares em animais e humanos.

\section{Neurogênese, angiogênese e sinaptogênese}

Dentre todos os efeitos da atividade física no cérebro, é a neurogênese o fenômeno neuroquímico mais associado ao impacto do exercício no SNC. O aumento da neurogênese hipocampal é um fenômeno robusto e claramente evidenciado 38-40;50-56. Estudos mostraram, no entanto, que parece não haver produção de novos neurônios ou células gliais em consequência do exercício, em outras regiões do cérebro, como por exemplo no bulbo olfatório ou zona subventricular ${ }^{57}$. Isso não se deve à falta de plasticidade na neurogênese olfatória. Na verdade, foi observado que o nascimento de novos neurônios olfatórios pode ser induzido por exposição a um ambiente rico em odores ${ }^{57}$. Em outras regiões, as evidências de neurogênese induzida pelo exercício ainda são controversas ${ }^{58-60}$. O exercício é capaz também de aumentar a proliferação de células da glia em camadas corticais superficiais, córtex motor ${ }^{(59)}$ e córtex pré-frontal de animais ${ }^{60}$. Ainda não se sabe até então o significado funcional desse aumento na gliogênese.

O exercício não só aumenta o número de novos neurônios, mas também influencia a morfologia de neurônios recém-nascidos, sugerindo que os efeitos do exercício nos novos neurônios é quantitativo e qualitativo. Utilizando uma estratégia de marcação retroviral, mostrou-se que neurônios recém-nascidos em consequência do exercício desenvolveram-se por meses no cérebro adulto ${ }^{61}$. Além disso foram observadas também alterações sinápticas nas mesmas regiões onde ocorreu neurogênese, sugerindo que as novas células têm papel funcional na integração do circuito neural. A neurogênese em consequência do exercício parece estar acompanhada ainda de um aumento no tamanho de espinhas dendríticas em áreas do hipocampo de corredores ${ }^{62}$ e na proliferação de células da glia (gliogênese) em camadas corticais superficiais, córtex motor e córtex pré-frontal de animais ${ }^{62}$.

A correlação entre exercício, neurogênese e memória também tem sido observada durante o envelhecimento normal. O exercício tem mostrado efeitos neuroprotetores contra o declínio cognitivo associado à idade e atrofia cerebral ${ }^{63,64}$ em cérebros adultos. Em estudos roedores e primatas não humanos, observou-se que a neurogênese cai a níveis baixos em consequência do envelhecimento e tem sido associada a déficits cognitivos ${ }^{65}$. A análise morfológica dos neurônios recém-nascidos em animais jovens e idosos mostrou não haver diferença entre os grupos no que diz respeito à morfologia dendrítica ${ }^{55}$.

\section{Inflamação e fatores periféricos de risco}

Por último, mecanismos sistêmicos apontam uma redução de fatores de risco periféricos em consequência do exercício. Um conceito emergente fundamental é que a saúde do cérebro e as funções cognitivas são moduladas pela interrelação de diversos fatores centrais e periféricos. Especificamente, a função cerebral depende da presença de fatores de risco periféricos para declínio cognitivo, incluindo hipertensão, hiperglicemia, resistência à insulina e dislipidemia - um amontoado de fatores que foram conceituados como "síndrome metabólica"66 Alguns dos vários aspectos dessa síndrome, os mais cruciais para a função cognitiva, são a hipertensão e a intolerância à glicose. Uma característica comum de muitas dessas condições é a inflamação sistêmica, que contribui para a maioria das condições na síndrome metabólica. Além disso, a inflamação sistêmica aumenta a inflamação no SNC e está associada ao declínio cognitivo ${ }^{19}$. Surpreendentemente, o exercício reduz todos os fatores de risco periféricos, melhorando a capacidade 
cardiovascular, equilíbrio lipídeo-colesterol, metabolismo energético, utilização de glicose, sensibilidade à insulina e inflamação ${ }^{19}$. Os efeitos centrais e periféricos do exercício, que resultam em melhoras da saúde cerebral e funções cognitivas, podem ser mediados por mecanismos comuns que convergem na modulação da sinalização de fatores de crescimento. Particularmente, o exercício pode induzir a sinalização de fatores de crescimento através do aumento direto destes fatores e da redução de citocinas pró-inflamatórias, que prejudicariam a sinalização de fatores neurotróficos ${ }^{38}$. Os efeitos do exercício na sinalização central e periférica de IGF-1 são um exemplo. Estudos mostram que a presença de citocinas pro-inflamatórias prejudica a transdução do sinal de IGF-1 e é um mecanismo de resistência à insulina ${ }^{19,38}$. O IGF-1 periférico é essencial para o metabolismo de glicose, manutenção do tecido, função cerebrovascular e, observou-se ainda que um baixo nível de IGF-1 traz riscos de prejúizo cognitivo ${ }^{19}$. O exercício aumenta o IGF-1 periférico, levando a melhoras na sua sinalização e na sensibilidade à insulina e, consequentemente, na saúde cerebral e funções cognitivas ${ }^{19}$. Além disso, citocinas pró-inflamatórias prejudicam a transdução do sinal de IGF-1 em neurônios ${ }^{19}$.

O exercício pode contra-atuar com os efeitos negativos desta inflamação através da recuperação do sinal de IGF-1, já que reduz a circulação de citocinas pró-inflamatórias. Outros estudos mostraram ainda que a redução na inflamação pelo exercício melhora a sinalização de BDNF. Citocinas pró-inflamatórias prejudicam a sinalização de BDNF nos neurônios, levando à uma condição conhecida como resistência à neurotrofina, que é conceitualmente similar à resistência à insulina ${ }^{66}$. Dados recentes indicam ainda que o exercício melhora a condição imune do cérebro, reduzindo, por exemplo a IL-1b (uma citocina pró-inflamatória) em modelos animais de Alzheimer e, desta forma, reduzindo a resposta inflamatória ao derrame ou infeccção periférica ${ }^{19}$. A Figura 2 resume os efeitos neurobiológicos do exercício.

Desse modo, o efeito do exercício no cérebro é único, no sentido de melhorar a saúde cerebral e as funções cognitivas através da redução de fatores de risco periféricos (indiretos) para declínio cognitivo e diretamente através das inúmeras alterações neuroquímicas mencionadas anteriormente. No entanto, apesar de evidências consistentes apoiarem a ideia de o exercício facilitar a memória e a aprendizagem em humanos e animais, há uma lacuna na literatura sobre quais tipos de aprendizagem podem melhorar com o exercício. Por exemplo, estudos em humanos sobre os efeitos do exercício na cognição têm observado alterações em tarefas associadas à área frontal ou funções executivas e estudos em animais avaliaram primariamente aprendizagem e plasticidade relacionada ao hipocampo. Pesquisas futuras devem refinar os estudos que investiguam os efeitos do exercício na cognição, de modo a melhorar a relevância tradução dos resultados em humanos.

\section{Exercício e saúde mental}

Nas últimas décadas, observou-se um progressivo aumento da prevalência de transtornos de humor na população adulta mundial. As estimativas mundias para a prevalência destes transtornos são significativamente altas, cerca de $20 \%{ }^{67}$. Isto significa que um grande número de pessoas experimentará algum tipo de transtorno de humor em algum período da vida, de maneira contínua ou recorrente. Algumas condições como estresse, ansiedade, depressão, fobias, transtornos compulsivos e pânico compreendem uma boa parte dos transtornos mentais observados. O estresse e a ansiedade excessiva são componentes-chave ou sintomas comuns em quase todas estas condições. O estresse é freqüente em adultos relativamente saudáveis e tem sido associado a conseqüências negativas na saúde, absenteísmo e redução na produtividade profissional ${ }^{67}$. Uma compreensão mais ampla da etiologia dos problemas relacionados ao estresse inclui uma multiplicidade de fatores: biológicos, psicológicos e sociais; todos mediados por fatores de risco e proteção ${ }^{68}$. O tratamento atual para transtornos de humor inclui intervenções terapêuticas e farmacológicas, ambas embasadas por grande quantidade de evidências empíricas através de estudos controlados ${ }^{(69)}$. No entanto, as pesquisas também sugerem que muitos indivíduos acometidos por estes transtornos acabam não procurando ajuda profissional, o que indica a necessidade da criação de

Nascidos para correr

a importância do exercício para a saúde do cérebro

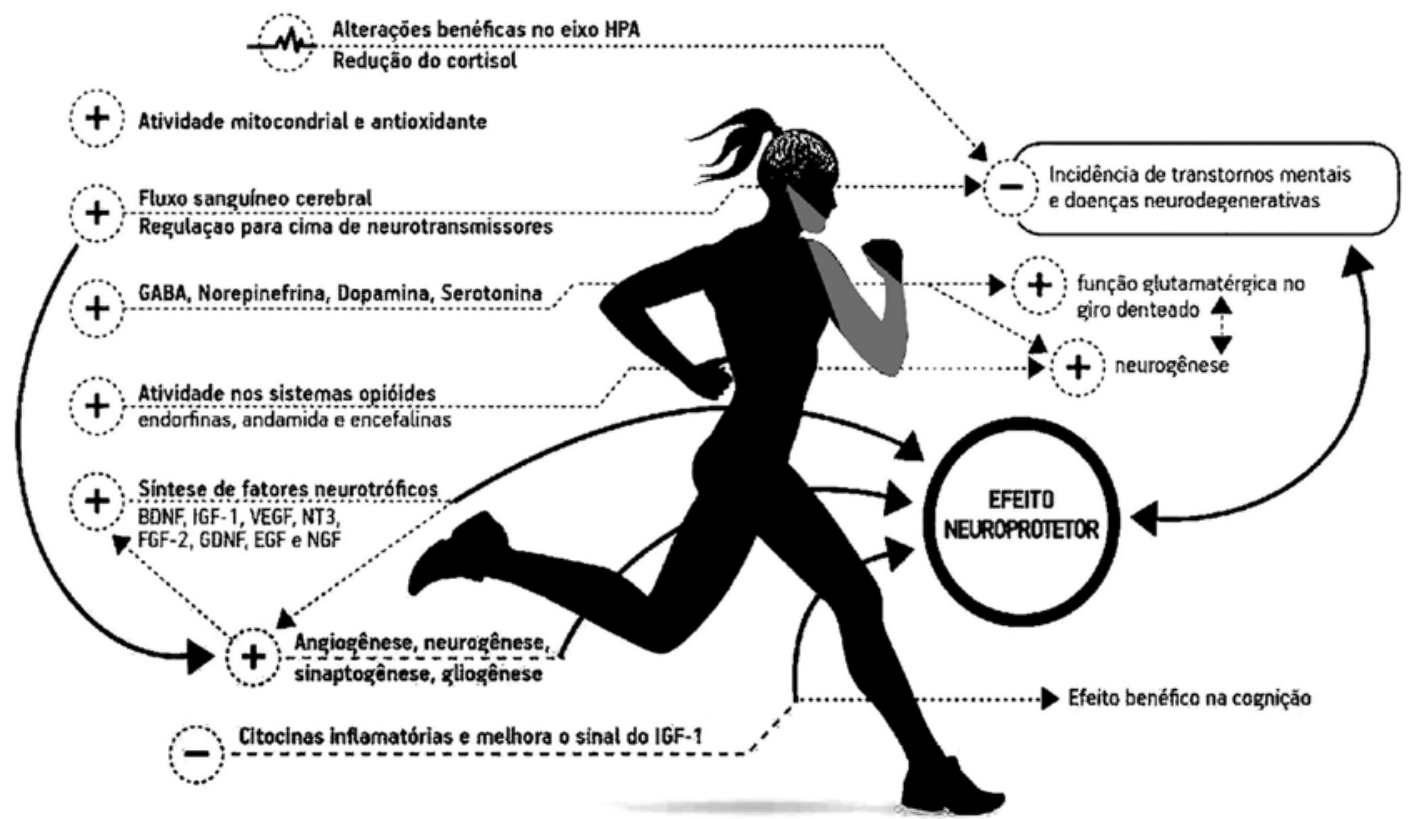

Figura 2. Efeitos do exercício no cérebro. 
auto-estratégias complementares apropriadas e confiáveis. Além disso, tanto pacientes quanto pesquisadores concordam em dois pontos: 1) não é satisfatório passar uma vida inteira fazendo uso de medicamentos e, 2) as estratégias terapêuticas tradicionais podem ser extremamente custosas se realizadas durante longo período. Ensaios clínicos vêm demonstrando que tanto drogas ansiolíticas quanto antidepressivas têm eficácia limitada em longo prazo ${ }^{68-70}$, causam dependência e sonolência, afetam cognição e memória e produzem disfunção sexual70.

Existe uma diversidade de abordagens terapêuticas tradicionais para o tratamento de transtornos mentais, mas muitas vezes os pacientes preferem procurar intervenções complementares por diversos motivos, tais como: efeitos adversos da medicação, falta de resposta ao tratamento, alto custo das psicoterapias, ou simplesmente preferência por alguma intervenção complementar específica.

Dentre as intervenções complementares comprovadamente eficazes no tratamento de transtornos mentais, estão o exercício físico e as práticas contemplativas, como o yoga e a meditação ${ }^{71,72}$. Embora com métodos e objetivos bem diversos, ambas as práticas têm em comum o efeito significativamente benéfico no sistema nervoso central e o fato de representarem para indivíduos saudáveis ou acometidos por transtornos mentais uma completa reformulação do estilo de vida e uma ação efetiva de promoção de saúde e prevenção de doenças.

\section{Exercício e transtornos de humor}

O tratamento farmacológico é ainda hoje o padrão ouro para tratamento de transtornos mentais, no entanto, os conhecidos efeitos adversos contribuem para possíveis falhas na conformidade e não aderência ao tratamento ${ }^{69}$. Deste modo, devem ser priorizadas estratégias que auxiliem na redução de medicações e internações e aumentem a qualidade de vida dos pacientes. Uma revisão mostrou que o exercício regular reduz os sintomas associados à transtornos mentais como depressão e doenças neurodegenerativos como Alzheimer e Parkinson ${ }^{18}$. Segundo os autores, a prática regular de exercício pode ser utilizada como coadjuvante eficaz no tratamento de diferentes transtornos mentais.

A depressão é um dos maiores problemas de saúde pública do mundo. Sua incidência é estimada em aproximadamente 20\% da população mundial ${ }^{73}$. Os sintomas incluem perda de peso, sentimento de culpa, ideação suicida, hipocondria, queixa de dores e psicose. Esses sintomas são mais acentuados em deprimidos idosos do que em jovens e contribuem para declínio cognitivo e do condicionamento cardiorrespiratório ${ }^{73}$. Além disso, somente cerca de 40\% dos pacientes depressivos respondem ao tratamento com psicofármacos ${ }^{74}$. Estudos mostram que o tratamento farmacológico reduz em cerca de $50 \%$ os sintomas relacionados, observados através de escalas de avaliação de depressão. No entanto, para a eventual remissão, faz-se necessária a utilização de outros métodos coadjuvantes de tratamento.

Ernst et al. ${ }^{75}$ sugerem que, dentre outros métodos, o exercício pode ser considerado eficaz no tratamento da depressão. Dentre os benefícios agudos e crônicos do exercício estão: melhora no condicionamento físico; diminuição da perda de massa óssea e muscular; aumento da força, coordenação e equilíbrio; redução da incapacidade funcional, da intensidade dos pensamentos negativos e das doenças físicas; e promoção da melhoria do bem-estar e do humor. Estudos que utilizaram o exercício como intervenção terapêutica na depressão concluíram que o grupo que praticava exercício apresentou maior recuperação e menor recaída que os outros e que quanto maior for o tempo gasto com exercícios, menores serão os níveis de depressão $0^{74-77}$. Outros estudos ${ }^{78}$ relacionaram ainda a diminuição da depressão a alterações no sistema imunológico.
Embora com resultados significativos no tratamento da depressão, os mecanismos pelos quais a atividade física proporciona efeitos antidepressivos são especulativos. Para tentar elucidá-los, faz-se necessário um entendimento da neurobiologia e neuropsicologia da depressão. No entanto, evidências emergentes sugerem que de fato o exercício tem efeitos preventivos e de tratamento na depressão. A depressão está relacionada ao declínio cognitivo e é considerado um fardo na saúde mundial ${ }^{74}$. Os efeitos terapêuticos do exercício na depressão tem sido estabelecidos em estudos humanos. Estudos randomizados demonstraram a eficácia do exercício aeróbico e de resistência (de 2 a 4 meses) como tratamento da depressão em indivíduos jovens e idosos ${ }^{73,74,78-80}$. Os benefícios são similares àqueles alcançados com antidepressivos e são dependentes da "dose": melhoras mais significativas estão associadas à maiores volumes de exercício ${ }^{79}$. Estudos observaram que tanto o exercício aeróbico moderado quanto o de resistência têm efeitos positivos nos sintomas depressivos ${ }^{74,76}$. Os autores concluíram que estes efeitos em idosos e indivíduos com sintomas depressivos moderados e que a eficácia do exercício depende do nível de aderência ao programa de exercícios e da combinação de exercício aeróbico moderado e exercício de resistência de alta intensidade .

Estudos de imagem observaram alterações no fluxo sangüíneo e no metabolismo do córtex pré-frontal; hiperatividade da região subgenual pré-frontal cortical; e aumento do metabolismo de glicose em várias regiões límbicas, especialmente na amígdala ${ }^{81}$, em função da depressão. Modificações na regulação do eixo HPA, como hipersecreção de cortisol, também estão relacionadas ao transtorno depressivo ${ }^{82,83}$. Além disso, alterações neurocognitivas como comprometimento na atenção, memória, velocidade de processamento, função executiva, emoção e tomada de decisão foram observadas em idosos depressivos ${ }^{82}$. Um dos fatores que podem explicar o déficit de memória na depressão é a alteração na atividade hipocampal, em consequência de hipercortisolemia, redução do BDNF e da neurogênese ${ }^{83}$.

Os efeitos do exercício no cérebro e, consequentemente na depressão, podem também estar relacionados ao aumento na liberação de monoaminas, como serotonina, dopamina e noradrenalina ${ }^{84,85}$. 0 processo da biossíntese de serotonina pode ocorrer pelo aumento de seu precursor triptofano no cérebro, influenciado pelo exercício ${ }^{84}$. A serotonina pode atenuar a formação de memórias relacionadas ao medo e diminuir as respostas a eventos ameaçadores através de projeções serotoninérgicas que partem do núcleo da rafe para o hipocampo ${ }^{84}$. 0 exercício também pode influenciar a síntese de dopamina em consequência do aumento nos níveis de cálcio no cérebro, através do aumento da atividade enzimática do sistema cálcio-calmodulina ${ }^{85}$. De modo geral e tendo em vista os benefícios físicos e psicológicos do exercício, é possivel concluir que sua prática por indivíduos depressivos é capaz de prevenir e reduzir significativamente sintomas depressivos. Há, no entanto, uma necessidade atual de pesquisas rigorosas, desenhadas para elucidar os mecanismos pelos quais o exercício promove a saúde mental.

\section{Exercício e doenças neurodegenerativas}

Diversos estudos têm demostrado que o exercício físico promove benefícios em portadores de doenças neurodegenerativas como Alzheimer ${ }^{86,87}$ e Parkinson ${ }^{88,89}$. Atualmente, o Alzheimer tornou-se a forma mais comum de demência em idosos, acometendo cerca de $50 \%$ de indivíduos acima de 90 anos $^{86}$. As alterações fisiopatológicas, como acúmulo de placas $\beta$-amilóide e emaranhados neurofibrilares, estão relacionadas à diminuição do volume cerebral, do número de neurônios, do número de sinapses e da extensão das ramificações dendríticas ${ }^{86,87}$. 
O exercício físico vem sendo apontado como uma eficaz estratégia não-farmacológica, cujo efeito protetor retarda o declínio cognitivo em consequência do envelhecimento ${ }^{90-92}$. Estudos em animais verificaram que o exercício de corrida voluntária aumenta a expressão de BNDF (fator neurotrófico derivado do cérebro), IGF1 (fator de crescimento semelhante a insulina tipo 1) e VEGF (Fator de crescimento endotelial vascular) ${ }^{44-49}$. Como já mencionado, o BNDF é um importante regulador da plasticidade sináptica e níveis diminuídos dessa proteína causam redução da plasticidade sináptica em áreas do cérebro afetadas durante o processo de envelhecimento ${ }^{11}$. Aleman e Torres-Alemán ${ }^{93}$ mostraram também que uma redução nos níveis de IGF-1 no cérebro pode contribuir para o declínio das funções cognitivas durante o envelhecimento. afirmam que maiores níveis de IGF1 em idosos saudáveis possuem correlação positiva com o aumento no volume do hipocampo e melhor desempenho verbal. Por outro lado, o exercício físico é capaz de regular para cima as concentrações de IGF-1 no cérebro.

Verificou-se também que o exercício aeróbico aumenta a complexidade dendrítica, o número de espinhas dendríticas no giro denteado ${ }^{26} \mathrm{e}$ a perfusão hipocampal em estado de repouso, tanto em roedores como em seres humanos jovens ${ }^{25}$, evidenciando a correlação entre angiogênise e neurogênese $e^{93}$. É possível que esse aumento do fluxo sanguíneo cerebral e maior metabolismo cerebral da glicose tenha relação com a degradação da proteína $\beta$-amilóide ${ }^{15}$, cujo acúmulo é responsável pela morte de neurônios colinérgicos no Alzheimer.

Além disso, o exercício influencia fatores de risco associados à demência, tais como a resistência à insulina, já que este em idosos está associado à redução do metabolismo de glicose no lobo temporal medial ${ }^{94,95}$. Assim, o exercício é capaz de melhorar a sensibilidade à insulina e contribuir potencialmente para a melhora da memória na doença ${ }^{96}$.

É possivel, deste modo, que existam diferentes mecanismos pelos quais o exercício atua como fator neuroprotetor para Alzheimer. Tais mecanismos incluem os efeitos mediados por fatores de risco cardiovascular (hipertensão, diabete, obesidade), e fatores neurobiológicos, como o aumento na concentração de neurotrofinas, essenciais para a manutenção das funções cognitivas e da plasticidade neural ${ }^{97}$.

Ainda não se sabe ao certo, no entanto, qual o melhor programa de exercícios para pacientes com Alzheimer O exercício aeróbico parece ser o mais indicado para essa população, já que está associado a uma melhora significativa na função cognitiva geral ${ }^{87}$. No entanto, pacientes acometidos por essa demência também apresentam baixa força e massa muscular ${ }^{98,99} \mathrm{Um}$ estudo no qual o programa compreendia um treinamento físico com exercício aeróbico, equilíbrio e treinamento de força, mostrou-se altamente eficaz na melhora da postura, do funcionamento motor e na redução do risco de queda ${ }^{98}$.

A doença de Parkinson é considerada a segunda doença neurodegenerativa mais comum atualmente, afetando cerca de 0,3\% da população em geral ${ }^{99}$. Considerada uma síndrome degenerativa e progressiva do sistema nervoso central, caracteriza-se pela perda dos neurônios dopaminérgicos da substância negra, provocando desordem dos movimentos, tremores em repouso, rigidez e bradicinesia ${ }^{99}$. Com a progressão da doença, outros problemas podem surgir, como instabilidade postural e disfunções da marcha, quedas e limitações funcionais progressivas, reduzindo substancialmente a qualidade de vida do paciente ${ }^{99}$.

A degeneração dopaminérgica nigro-estriatal é um dos principais mecanismos da doença, cujos déficits atingem, principalmente, mecanismos motores ${ }^{99,100}$. Além destes, outros, como os circuitos serotoninérgicos, noradrenérgicos e colinérgicos, também são afetados na doença de Parkinson, e contribuem para as disfunções cognitivas presentes em alguns $\operatorname{casos}^{101}$.

O conhecimento atual sobre os mecanismos envolvidos no efeito neuroprotetor contra o Parkinson, baseia-se em resultados obtidos em modelos animais ${ }^{86}$. Tem sido demonstrado que o exercício tem efeito significativo na função dopaminérgica, de modo a estimular a expressão de fatores neurotróficos e angiogênese ${ }^{100}$. Em resposta ao exercício aeróbico, observa-se um aumento na concentração de dopamina, assim como na sensibilidade de seus receptores ${ }^{100}$. Mais especificamente, verifica-se uma redução da alteração dos neurônios dopaminérgicos na substância negra, o que contribui para a reconstituição da função dos gânglios da base, envolvidos no comando do movimento, mecanismos adaptativos da dopamina e neurotransmissão glutamatérgica ${ }^{102}$ e tal ação está relacionada ao aumento da concentração de BDNF ${ }^{86}$.

Estudos sugerem ainda que a prática de atividade física pode impedir o desenvolvimento da doença ${ }^{103-108}$. Rabadi ${ }^{109}$ verifica que o risco de desenvolver Parkinson parece ser inversamente proporcional à quantidade de atividade física praticada ao longo da vida. sugerem também que as pessoas que praticam exercícios durante a idade adulta e no fim da vida têm um risco 40\% menor de desenvolver a doença do que as pessoas que permaneceram inativas durante os mesmos períodos. Além disso, os efeitos positivos dessa prática sobre os componentes cognitivos e automáticos de controle motor em indivíduos parkinsonianos é resultado de mecanismos de neuroplasticidade que envolvem conexões sinápticas de redes neurais ${ }^{86}$. No que concerne à função motora de indivíduos parkinsonianos, há evidências de que o treinamento de força melhora de modo significativo a velocidade de força muscular ${ }^{104}$.

Diante disso, evidencia-se a importância do exercício físico na prevenção e intervenção terapêutica em doença neurodegenerativa, atenuando e até limitando a progressão destas doenças.

Ainda sobre o efeito neuroprotetor do exercício, esta influência pode também estra associada à redução de dano cerebral e ao atraso no estabelecimento e declínio de doenças neurodegenerativas. Estudos mostram, por exemplo, que a participação em um programa de exercícios após acidente vascular cerebral (AVC) acelera a reabilitação funcional ${ }^{109}$. Modelos animais de isquemia (oclusão da arteria medial cerebral) sugerem que terapias cardiovasculares sozinhas podem reduzir dano associado ao AVC e melhorar a recuperação ${ }^{109}$. Um objetivo essencial de pesquisas futuras sobre reabilitação é definir o tipo, intensidade e frequência das intervenções com exercício. Além dos benefícios do exercício na reabilitação pós-AVC, estudos retrospectivos sugerem que a prática de atividades físicas retarda o aparecimento e reduz risco para Alzheimer, Parkinson e Huntington, podendo ainda reduzir o declínio funcional após início da neurodegeneração ${ }^{96,109}$. Indívíduos com Alzheimer mostram melhoras em escalas de atividades cotidianas, em testes cognitivos, sintomas depressivos e funções físicas, comparados aos que não se exercitam, cujas funções continuam a declinar ${ }^{110}$. Estudos com pacientes com Parkinson mostram que o exercício aeróbico melhora a iniciação do movimento e capacidade aeróbica ${ }^{107}$, assim como o desempenho de atividades diárias.

As altas taxas de inatividade física, no entanto, tornam difícil a aquisição integral de todos os benefícios do exercicio supracitados. Quanto maior o volume de atividade física não-extenuante, maiores os efeitos ${ }^{111,112}$. Ekkekakis e Petruzzello ${ }^{111}$ observaram que a resposta aguda afetiva ao exercício é fator determinante na aderência, pois são utilizadas vias cognitivas como o aumento da autonomia percebida e auto-eficácia interoceptiva e o acúmulo de lactato sanguíneo. Isto é, a relação entre exercício (aeróbico) e estado afetivo induzido pode predizer com certa acurácia a aderência a um programa de exercícios, fator essencial para a condição terapêutica desta prática. De acordo com ${ }^{113}$, há 
uma relação de $U$ invertido entre a intensidade do exercício aeróbico e estado afetivo. Esta teoria sugere que a intensidade ótima do exercício, aquela que produz respostas afetivas mais positivas, seria a moderada, próxima ao limiar ventílatório $\left(\sim 65 \% \mathrm{VO}_{2} \text { máx. }\right)^{113}$. Sugere-se ainda que intensidades acima deste limiar são percebidas como ameaçadoras pela maioria dos indivíduos e tendem a gerar estados afetivos negativos, prejudicando a aderência ao programa de exercícios ${ }^{113}$. Uma meta-análise recente concluiu que exercícios de baixa intensidade com duração de 35 minutos ou menos são capazes de induzir fortemente estados afetivos positivos ${ }^{114}$. Há ainda evidências que sugerem que resultados comportamentais positivos relacionados à aderência podem ocorrem quando indivíduos se exercitam em intensidades auto-selecionadas ${ }^{114}$.

Deste modo, conclui-se que, a fim se de obter o potencial máximo dos efeitos do exercício, deve-se levar em consideração primariamente a prescrição ideal (volume versus intensidade) e a individualidade biológica do praticante. Mas, de modo geral, os melhores resultados da relação entre duraçãolintesidade do exercício e qualidade de vida, assim como efeitos nas funções cognitivas, apontam para intensidades moderadas de exercício aeróbico.

\section{CONSIDERAÇÕES FINAIS}

A área da Neurociência do Exercício é relativamente nova, mas décadas de estudos experimentais e longitudinais em humanos e animais vêm demonstrando que os efeitos do exercício no cérebro são significativamente positivos, representando uma poderosa estratégia terapêutica em saúde mental. Seja através de efeitos diretos no sistema nervoso central como a proliferação de novos neurônios, o aumento de fatores neurotróficos, gliogênese, sinaptogênese e regulação de sistemas de neurotransmissão e neuromodulação ou à partir de efeitos indiretos como a redução da inflamação sistêmica, o impacto do exercício no cérebro é único no sentido de melhorar a saúde mental, reduzir o declínio de massa cinzenta associado à idade e melhorar as funções cognitivas. Além disso, o exercício tem se mostrado uma ferramenta altamente eficaz no tratamento de transtornos mentais como depressão, ansiedade e doenças neurodegenerativas. Dados os recentes avanços na presente temática e o potencial terapêutico e econômico do exercício na população em geral, espera-se que pesquisas futuras correlacionando pesquisas básicas à variáveis psicológicas e estudos de imagem possam elucidar melhor os mecanismos pelos quais o exercício melhora a saúde cerebral.

\section{AGRADECIMENTOS}

Os autores agradecem à Fundação de Apoio à Pesquisa e Inovação Tecnológica do Estado de Sergipe (FAPITEC) pelo apoio financeiro à pesquisa e elaboração do projeto.

Todos os autores declararam não haver qualquer potencial conflito de interesses referente a este artigo.

CONTRIBUIÇÕES DOS AUTORES: Cada autor contribuiu individual e significativamente para o desenvolvimento deste manuscrito. CV (0000-0002-0809-3964)*: redação, conteúdo intelectual e revisão do manuscrito; RSG (0000-0001-6032-0549)* e LPG (0000-0002-3851-5005)*: redação de parte da temática do manuscrito; AB (0000-00020106-7210)*: elaboração de imagens e redação de parte da temática do manuscrito; AB (0000-0003-2403-8081)*: sugestões e revisão do manuscrito; EHMD (0000-00030981-8020)*: sugestões e revisão geral do manuscrito. Todos os autores aprovaram a versão final do manuscrito. *ORCID (Open Researcher and Contributor ID).

\section{REFERÊNCIAS}

1. Bramble DM, Lieberman DE. Endurance running and the evolution of Homo. Nature. 2004:432(7015):345-52.

2. Lieberman DE. Understanding apes. In: The story of the human body: evolution, health and disease. New York: Pantheon Press; 2013.

3. Lieberman DE. Much depends on dinner. The Story of the Human Body: Evolution, Health and Disease. New York: Pantheon Press, 2013.

4. Lieberman DE. Four legs good, two legs fortuitous: brains, brawn and the evolution of human bipedalism. In: Losos JB, editor. In the light of evolution. Greenwood Village, CO: Roberts and Company; 2010. p. 55-71.

5. Booth FW, Roberts CK, Laye MJ. Lack of exercise is a major cause of chronic diseases. Compr Physiol. 2012;2(2):1143-211.

6. Biddle SJ, Fox KR, Boutcher SH. Physical activity and psychological well-being. London: Routledge; 2000

7. Chang YK, Nien YH, Tsai CL, Etnier JL. Physical activity and cognition in older adults: the potential of Tai Chi Chuan. J Aging Phys Act. 2010;18(4):451-72.

8. Blumenthal JA, Babyak MA, Doraiswamy PM, Watkins L, Hoffman BM, Barbour KA, et al. Exercise and pharmacotherapy in the treatment of major depressive disorder. Psychosom Med. 2007;69(7):587-96.

9. Duzel E, van Praag H, Sendtner M. Can physical exercise in old age improve memory and hippocampal function? Brain. 2016;139(Pt 3):662-73

10. Mello MT, Boscolo RA, Esteves AM, Tufilk SO. Exercício físico e os aspectos psicobiológicos. Rev Bras Med Esporte. 2005;11(3):195-9.

11. Lista I, Sorrentino G. Biological mechanisms of physical activity in preventing cognitive decline. Cell Mol Neurobiol. 2010;30(4):493-503.

12. Chaddock L, Erickson KI, Prakash RS, Kim JS, Voss MW, Vanpatter M, et al. A neuroimaging investigation of the association between aerobic fitness, hippocampal volume, and memory performance in preadolescent children. Brain Res. 2010;1358:172-83.

13. Jin K, Galvan V, Xie L, Mao XO, Gorostiza OF, Bredesen DE, et al. Enhanced neurogenesis in Alzheimer's disease transgenic (PDGF-APPSw, Ind) mice.Proc Natl Acad Sci U S A. 2004;101(36):13363-7.

14. Zhao C, Teng EM, Summers RG Jr, Ming GL, Gage FH. Distinct morphological stages of dentate granule neuron maturation in the adult mouse hippocampus. J Neurosci. 2006;26(1):3-11.

15. Matta Mello Portugal E, Cevada T, Sobral Monteiro-Junior R, Teixeira Guimarães T, da Cruz Rubini E, Lattari E, et al. Neuroscience of exercise: from neurobiology mechanisms to mental health. Neuropsychobiology. 2013;68(1):1-14.

16. Leem YH, Lee Yl, Son HJ, Lee SH. Chronic exercise ameliorates the neuroinflammation in mice carrying NSE/htau23. Biochem Biophys Res Commun. 2011;406(3):359-65.

17. Kronenberg G, Bick-Sander A, BunkE, WolfC, Ehninger D, Kempermann G. Physical exercise prevents age-related decline in precursor cell activity in the mouse dentate gyrus. Neurobiol Aging. 2006;27(10):1505-13.

18. Deslandes A, Moraes H, Ferreira C, Veiga H, Silveira H, Mouta R, et al. Exercise and mental health: many reasons to move. Neuropsychobiology. 2009;59(4):191-8.

19. Kim B, Feldman EL. Insulin resistance as a key link for the increased risk of cognitive impairment in the metabolic syndrome. Exp Mol Med. 2015;47:e149.

20. Laugero KD. A new perspective on glucocorticoid feedback: relation to stress,carbohydrate feeding and feeling better. J Neuroendocrinol. 2001;13(9):827-35.

21. Radak Z, Chung HY, Goto S. Systemic adaptation to oxidative challenge induced by regular exercise Free Radic Biol Med. 2008;44(2):153-9.

22. Powers SK, Talbert EE, Adhihetty PJ. Reactive oxygen and nitrogen species as intracellular signals in skeletal muscle. J Physiol. 2011;589(Pt 9):2129-38.

23. Cheik NC, Reis IT, Heredia RA, Ventura ML, Tufik S, Antunes HK, et al. Efeitos do exercício físico e da atividade física na depressão e ansiedade em indivíduos idosos. Rev Bras Ci e Mov. 2003;11(3):45- 52.

24. Encinas JM, Vaahtokari A, Enikolopov G. Fluoxetine targets early progenitor cells in the adult brain. Proc Natl Acad Sci U S A. 2006;103(21):8233-8.

25. Maass A, Düzel S, Brigadski T, Goerke M, Becke A, Sobieray U, et al. Relationships of peripheral IGF-1, VEGF and BDNF levels to exercise-related changes in memory, hippocampal perfusion and volumes in older adults. Neuroimage. 2016;131:142-54.

26. Eadie BD, Redila VA, Christie BR. Voluntary exercise alters the cytoarchitecture of the adult dentate gyrus by increasing cellular proliferation, dendritic complexity, and spine density. J Comp Neurol. 2005 May 23;486(1):39-47.

27. Tong L, Shen H, Perreau VM, Balazs R, Cotman CW. Effects of exercise on gene-expression profile in the rat hippocampus. Neurobiol Dis. 2001;8(6):1046-56.

28. Molteni R, Ying Z, Gómez-Pinilla F. Differential effects of acute and chronic exercise on plasticity-related genes in the rat hippocampus revealed by microarray. Eur J Neurosci. 2002;16(6):1107-16.

29. Schlett K. Glutamate as a modulator of embryonic and adult neurogenesis. Curr Top Med Chem. 2006;6(10):949-60.

30. Neeper SA, Gómez-Pinilla F, Choi J, Cotman C. Exercise and brain neurotrophins. Nature. 1995;373(6510):109.

31. Sforzo GA, Seeger TF, Pert CB, Pert A, Dotson CO. In vivo opioid receptor occupation in the rat brain following exercise. Med Sci Sports Exerc. 1986;18(4):380-4.

32. Eisch AJ, Barrot M, Schad CA, Self DW, Nestler EJ. Opiates inhibit neurogenesis in the adult rat hippocampus. Proc Natl Acad Sci U S A. 2000;97(13):7579-84.

33. Harburg GC, Hall FS, Harrist AV, Sora I, Uhl GR, Eisch AJ. Knockout of the mu opioid receptor enhances the survival of adult-generated hippocampal granule cell neurons. Neuroscience. 2007;144(1):77-87.

34. Persson Al, Naylor AS, Jonsdottir IH, Nyberg F, Eriksson PS, Thorlin T. Differential regulation of hippocampal progenitor proliferation by opioid receptor antagonists in running and non-running spontaneously hypertensive rats. Eur J Neurosci. 2004;19(7):1847-55.

35. Narita M, Kuzumaki N, Miyatake M, Sato F, Wachi H, Seyama Y, et al. Role of delta-opioid receptor function in neurogenesis and neuroprotection. J Neurochem. 2006;97(5):1494-505.

36. KoehI M, Meerlo P, Gonzales D, Rontal A, Turek FW, Abrous DN. Exercise-induced promotion of hippocampal cell proliferation requires beta-endorphin. FASEB J. 2008;22(7):2253-62.

37. Fuss J, Steinle J, Bindila L, Auer MK, Kirchherr H, Lutz B, et al. A runner's high depends on cannabinoid receptors in mice. Proc Natl Acad Sci U S A. 2015;112(42):13105-8.

38. Cotman CW, Berchtold NC, Christie LA. Exercise builds brain health: key roles of growth factor cascades and inflammation. Trends Neurosci. 2007;30(9):464-72.

39. Russo-Neustadt A, Beard RC, Cotman CW. Exercise, antidepressant medications, and enhanced brain derived neurotrophic factor expression. Neuropsychopharmacology. 1999;21(5):679-82. 
40. Palmer TD, Willhoite AR, Gage FH. Vascular niche for adult hippocampal neurogenesis. J Comp Neurol. 2000;425(4):479-94

41. Chen H, Zhang SM, Schwarzschild MA, Hernán MA, Ascherio A. Physical activity and the risk of Parkinson disease. Neurology. 2005;64(4):664-9.

42. Winter B, Breitenstein C, Mooren FC, Voelker K, Fobker M, Lechtermann A, et al. High impact running improves learning. Neurobiol Learn Mem. 2007;87(4):597-609.

43. Isaacs KR, Anderson BJ, Alcantara AA, Black JE, Greenough WT. Exercise and the brain: angiogenesis in the adult rat cerebellum after vigorous physical activity and motor skill learning. J Cereb Blood Flow Metab. 1992;12(1):110-9.

44. Cao L, Jiao X, Zuzga DS, Liu Y, Fong DM, Young D, et al.VEGF links hippocampal activity with neurogenesis, learning and memory. Nat Genet. 2004;36(8):827-35.

45. Aberg MA, Aberg ND, Hedbäcker H, Oscarsson J, Eriksson PS. Peripheral infusion of IGF-I selectively induces neurogenesis in the adult rat hippocampus. J Neurosci. 2000;20(8):2896-903.

46. Shen Q, Goderie SK, Jin L, Karanth N, Sun Y, Abramova N, et al. Endothelial cells stimulate self-renewal and expand neurogenesis of neural stem cells. Science. 2004;304(5675):1338-40.

47. Thored P, Wood J, Arvidsson A, Cammenga J, Kokaia Z, Lindvall O. Long-term neuroblast migration along blood vessels in an area with transient angiogenesis and increased vascularization after stroke. Stroke. 2007;38(11):3032-9.

48. De Rossi P, Harde E, Dupuis JP, Martin L, Chounlamountri N, Bardin M, et al. A critical role for VEGF and VEGFR2 in NMDA receptor synaptic function and fear-related behavior. Mol Psychiatry. 2016;21(12):1768-1780.

49. Lichtenwalner RJ, Forbes ME, Bennett SA, Lynch CD, Sonntag WE, Riddle DR. Intracerebroventricular infusion of insulin-like growth factor-l ameliorates the age-related decline in hippocampal neurogenesis. Neuroscience. 2001;107(4):603-13.

50. Colcombe SJ, Erickson KI, Raz N, Webb AG, Cohen NJ, McAuley E, et al. Aerobic fitness reduces brain tissue loss in aging humans. J Gerontol A Biol Sci Med Sci. 2003;58(2):176-80.

51. van Praag H, Shubert T, Zhao C, Gage FH. Exercise enhances learning and hippocampal neurogenesis in aged mice. J Neurosci. 2005;25(38):8680-5.

52. Fabel K, Fabel K, Tam B, Kaufer D, Baiker A, Simmons N, et al. VEGF is necessary for exercise-induced adult hippocampal neurogenesis. Eur J Neurosci. 2003;18(10):2803-12

53. Trejo JL, Carro E, Torres-Aleman I. Circulating insulin-like growth factor I mediates exercise-induced increases in the number of new neurons in the adult hippocampus. J Neurosci. 2001;21(5):1628-34.

54. Vivar C, Potter MC, van Praag H. All about running: synaptic plasticity, growth factors and adult hippocampal neurogenesis. Curr Top Behav Neurosci. 2013;15:189-210.

55. van Praag H, Schinder AF, Christie BR, Toni N, Palmer TD, Gage FH. Functional neurogenesis in the adult hippocampus. Nature. 2002 Feb 28;415(6875):1030-4.

56. Kitamura T, Mishina M, Sugiyama $\mathrm{H}$. Enhancement of neurogenesis by running wheel exercises is suppressed in mice lacking NMDA receptor epsilon 1 subunit. Neurosci Res. 2003;47(1):55-63.

57. Rochefort C, Gheusi G, Vincent JD, Lledo PM. Enriched odor exposure increases the number of newborn neurons in the adult olfactory bulb and improves odor memory. J Neurosci. 2002;22(7):2679-89.

58. Gould E, Reeves AJ, Graziano MS, Gross CG. Neurogenesis in the neocortex of adult primates. Science. 1999;286(5439):548-52.

59. Dayer AG, Cleaver KM, Abouantoun T, Cameron HA. New GABAergic interneurons in the adult neocortex and striatum are generated from different precursors. J Cell Biol. 2005;168(3):415-27.

60. Kornack DR, Rakic P. Cell proliferation without neurogenesis in adult primate neocortex. Science. 2001;294(5549):2127-30.

61. Bronzino JD, Abu-Hasaballah K, Austin-LaFrance RJ, Morgane PJ. Maturation of long-term potentiation in the hippocampal dentate gyrus of the freely moving rat. Hippocampus. 1994;4(4):439-46.

62. Ehninger D, Kempermann G. Regional effects of wheel running and environmental enrichment on cell genesis and microglia proliferation in the adult murine neocortex. Cereb Cortex. 2003;13(8):845-51.

63. Kramer AF, Hahn S, Cohen NJ, Banich MT, McAuley E, Harrison CR, et al. Ageing, fitness and neurocognitive function. Nature. 1999;400(6743):418-9.

64. Yaffe K, Barnes D, Nevitt M, Lui LY, Covinsky K. A prospective study of physical activity and cognitive decline in elderly women: women who walk. Arch Intern Med. 2001;161(14):1703-8.

65. Leuner B, Kozorovitskiy Y, Gross CG, Gould E. Diminished adult neurogenesis in the marmoset brain precedes old age. Proc Natl Acad Sci U S A. 2007;104(43):17169-73.

66. Willette AA, Bendlin BB, Starks EJ, Birdsill AC, Johnson SC, Christian BT, et al. Association of Insulin Resistance With Cerebral Glucose Uptake in Late Middle-Aged Adults at Risk for Alzheimer Disease. JAMA Neurol. 2015;72(9):1013-20.

67. Manzoni GM, Pagnini F, Castelnuovo G, Molinari E. Relaxation training for anxiety: a ten-years systematic review with meta-analysis. BMC Psychiatry. 2008;8:41

68. Somers JM, Goldner EM, Waraich P, Hsu L. Prevalence and incidence studies of anxiety disorders: a systematic review of the literature. Can J Psychiatry. 2006;51(2):100-13.

69. Youngstedt SD, Kripke DF. Does bright light have an anxiolytic effect? - na open trial. BMC Psychiatry. 2007;7:62

70. Buffett-Jerrott SE, Stewart SH. Cognitive and sedative effects of benzodiazepine use. Curr Pharm Des. 2002;8(1):45-58

71. Balasubramaniam M, Telles S, Doraiswamy PM. Yoga on our minds: a systematic review of yoga for neuropsychiatric disorders. Front Psychiatry. 2013;3:117.

72. Struzik L, Vermani M, Coonerty-Femiano A, Katzman MA. Treatments for generalized anxiety disorder. Expert Rev Neurother. 2004;4(2):285-94.

73. Antunes HK, Stella SG, Santos RF, Bueno OF, de Mello MT. Depression, anxiety and quality of life scores in seniors after an endurance exercise program. Ver Bras Psiquiatr. 2005;27(4):266-71.

74. Frazer CJ, Christensen H, Griffiths KM. Effectiveness of treatments for depression in older people. Med J Aust. 2005;182(12):627-32

75. Ernst C, Olson AK, Pinel JP, Lam RW, Christie BR. Antidepressant effects of exercise: evidence for an adult-neurogenesis hypothesis? J Psychiatry Neurosci. 2006;31 (2):84-92

76. Fountoulakis KN, O'Hara R, lacovides A, Camilleri CP, Kaprinis S, Kaprinis G, et al. Unipolar late-onset depression: A comprehensive review. Ann Gen Hosp Psychiatry. 2003;2(1):11.
77. Kohut ML, LeeW, Martin A, Arnston B, Russell DW, Ekkekakis P, et al. The exercise-induced enhancement of influenza immunity is mediated in part by improvements in psychosocial factors in older adults. Brain Behav Immun. 2005;19(4):357-66.

78. Haboush A, Floyd M, Caron J, LaSota M, Alvarez K. Ballroom dance lessons for geriatric depression: an exploratory study. Arts Psychother. 2006;33(2):89-97.

79. Lawlor DA, Hopker SW. The effectiveness of exercise as an intervention in the management of depression: systematic review and meta-regression analysis of randomised controlled trials. BMJ. 2001;322(7289):763-7.

80. Hurley BF, Hanson ED, Sheaff AK. Strength training as a countermeasure to aging muscle and chronic disease. Sports Med. 2011;41(4):289-306.

81. Davidson RJ, Lewis DA, Alloy LB, Amaral DG, Bush G, Cohen JD, et al. Neural and behavioral substrates of mood and mood regulation. Biol Psychiatry. 2002;52(6):478-502.

82. Barden N. Implication of the hypothalamic-pituitary-adrenal axis in the physiopathology of depression J Psychiatry Neurosci. 2004;29(3):185-93

83. Joca SR, Padovan CM, Guimarães FS. Stress, depression and the hippocampus. Rev Bras Psiquiatr. 2003;25 Suppl 2:46-51

84. Weicker H, Strüder HK. Influence of exercise on serotonergic neuromodulation in the brain. Amino Acids. 2001;20(1):35-47.

85. Sutoo D, Akiyama K. Regulation of brain function by exercise. Neurobiol Dis. 2003;13(1):1-14

86. Paillard T, Rolland Y, de Souto Barreto P. Protective effects of physical exercise in Alzheimer's Disease and Parkinson's Disease: a narrative review. J Clin Neurol. 2015;11(3):212-9.

87. Teri L, Gibbons LE, McCurry SM, Logsdon RG, Buchner DM, Barlow WE, et al. Exercise plus behavioral management in patients with Alzheimer disease: a randomized controlled trial. JAMA. 2003;290(15):2015-22

88. Petzinger GM, Fisher BE, McEwen S, Beeler JA, Walsh JP, Jakowec MW. Exercise-enhanced neuroplasticity targeting motor and cognitive circuitry in Parkinson's disease. Lancet Neurol. 2013;12(7):716-26.

89. Petzinger GM, Fisher BE, Van Leeuwen JE, Vukovic M, Akopian G, Meshul CK, et al. Enhancing neuroplasticity in the basal ganglia: the role of exercise in Parkinson's disease. Mov Disord. 2010;25 Suppl 1:S141-5.

90. Jorm AF, Jolley D. The incidence of dementia: a meta-analysis. Neurology.1998:51(3):728-33.

91. Swerdlow RH. Pathogenesis of Alzheimer's disease. Clin Interv Aging. 2007;2(3):347-59.

92. Zigmond MJ, Cameron JL, Hoffer BJ, Smeyne RJ. Neurorestoration by physical exercise: moving forward. Parkinsonism Relat Disord. 2012;18 Suppl 1:S147-50.

93. Aleman A, Torres-Alemán I. Circulating insulin-like growth factor I and cognitive function: neuromodulation throughout the lifespan. Prog Neurobiol. 2009;89(3):256-65.

94. Brown BM, Peiffer JJ, Taddei K, Lui JK, Laws SM, Gupta VB, et al. Physical activity and amyloid- $\beta$ plasma and brain levels: results from the Australian Imaging, Biomarkers and Lifestyle Study of Ageing. Mol Psychiatry. 2013;18(8):875-81.

95. Cooper C, Sommerlad A, Lyketsos CG, Livingston G. Modifiable predictors of dementia in mild cognitive impairment: a systematic review and meta-analysis. Am J Psychiatry. 2015;172(4):323-34.

96. Lucas SJ, Cotter JD, Brassard P, Bailey DM. High-intensity interval exercise and cerebrovascular health: curiosity, cause, and consequence. J Cereb Blood Flow Metab. 2015;35(6):902-11.

97. Vaisberg M, Mello MT. Exercícios na saúde e na doença. Barueri, SP: Manole; 2010.

98. Suttanon P, Hill KD, Said CM, Williams SB, Byrne KN, LoGiudice D, et al. Feasibility, safety and preliminary evidence of the effectiveness of a home-based exercise programme for older people with Alzheimer's disease: a pilot randomized controlled trial. Clin Rehabil. 2013;27(5):427-38.

99. Ibañez V. Les maladies neuro-dégénératives: problmes cliniques. Med Nucl. 2005;29(4):213-9.

100.Speelman AD, van de Warrenburg BP, van Nimwegen M, Petzinger GM, Munneke M, Bloem BR. How might physical activity benefit patients with Parkinson disease? Nat Rev Neurol. 2011;7(9):528-34.

101.Zgaljardic DJ, Foldi NS, Borod JC. Cognitive and behavioral dysfunction in Parkinson's disease: neurochemical and clinicopathological contributions. J Neural Transm (Vienna). 2004;111(10-11):1287-301.

102.Alonso-Frech F, Sanahuja JJ, Rodriguez AM. Exercise and physical therapy in early management of Parkinson disease. Neurologist. 2011;17(6 Suppl 1):S47-53

103.Xu Q, Park Y, Huang X, Hollenbeck A, Blair A, Schatzkin A, et al. Physical activities and future risk of Parkinson disease. Neurology. 2010;75(4):341-8..

104.Lima LO, Scianni A, Rodrigues-de-Paula F. Progressive resistance exercise improves strength and physical performance in people with mild to moderate Parkinson's disease: a systematic review. Physiother. 2013;59(1):7-13.

105.Earhart GM, Williams AJ. Treadmill training for individuals with Parkinson disease. Phys Ther 2012;92(7):893-7.

106. Esculier JF, Vaudrin J, Bériault P, Gagnon K, Tremblay LE. Home-based balance training programme using Wii Fit with balance board for Parkinsons's disease: a pilot study. J Rehabil Med. 2012;44(2):144-50

107.Bergen JL, Toole T, Elliott RG 3rd, Wallace B, Robinson K, Maitland CG. Aerobic exercise intervention improves aerobic capacity and movement initiation in Parkinson's disease patients. NeuroRehabilitation. 2002;17(2):161-8

108. Weuve J, Kang JH, Manson JE, Breteler MM, Ware JH, Grodstein F. Physical activity, including walking, and cognitive function in older women. JAMA. 2004;292(12):1454-61.

109. Rabadi MH. Randomized clinical stroke rehabilitation trials in 2005. Neurochem Res. 2007;32(4-5):807-21.

110.Heyn P, Abreu BC, Ottenbacher KJ. The effects of exercise training on elderly persons with cognitive impairment and dementia: a meta-analysis. Arch Phys Med Rehabil. 2004;85(10):1694-704.

111.Ekkekakis P, Petruzzello SJ. Acute aerobic exercise and affect: current status, problems and prospects regarding dose-response. Sports Med. 1999;28(5):337-74

112.Ekkekakis P. Pleasure and displeasure from the body: perspectives from exercise. Cogn Emot. 2003;17(2):213-39.

113.Ekkekakis P, Hall EE, Petruzzello SJ. The relationship between exercise intensity and affective responses demystified: to crack the 40-year-old nut, replace the 40-year-old nutcracker! Ann Behav Med. 2008;35(2):136-49.

114.Ekkekakis P. Let them roam free? Physiological and psychological evidence for the potential of self-selected exercise intensity in public health. Sports Med. 2009;39(10):857-88. 